\title{
An isoperimetric inequality related to a Bernoulli problem*
}

\author{
Daniel Daners $\quad$ Bernd Kawohl
}

January 28, 2010

\begin{abstract}
Given a bounded domain $\Omega$ we look at the minimal parameter $\Lambda(\Omega)$ for which a Bernoulli free boundary value problem for the $p$-Laplacian has a solution minimising an energy functional. We show that amongst all domains of equal volume $\Lambda(\Omega)$ is minimal for the ball. Moreover, we show that the inequality is sharp with essentially only the ball minimising $\Lambda(\Omega)$. This resolves a problem related to a question asked in [Flucher et al., J. Reine Angew. Math. 486 (1997), 165-204.].
\end{abstract}

Mathematics Subject Classification (2000). 35R35, 49R05

Keywords. isoperimetric inequality, Bernoulli problem, free boundary, symmetrisation

\section{Motivation and Result}

For given $\lambda>0$ consider the following Bernoulli type free boundary problem

$$
\begin{aligned}
\Delta v=0 & & \text { in } \Omega \backslash D, \\
v=0 & & \text { on } \partial \Omega, \\
v \equiv 1 & & \text { on } D, \\
|\nabla v|=\lambda & & \text { on } \partial D,
\end{aligned}
$$

on a given bounded open set $\Omega \subset \mathbb{R}^{N}$, where $D \subset \Omega$ is an unknown closed set. Such free boundary value problems originally arose from two dimensional

*This research is part of the ESF program "Global and geometric aspects of nonlinear partial differential equations (GLOBAL)". 
flows (see $[2,7]$ ), but also have applications to heat flows or electro-chemical machining (see the references in [4]).

It was shown in [1, Section 1.3] that some solutions to (1.1) can be obtained as non-trivial minimisers of the the functional

$$
J_{\lambda}(u):=\int_{\Omega}|\nabla u(x)|^{2} d x+\lambda^{2}|\{u<1\}|
$$

over all $u \in H_{0}^{1}(\Omega)$ (Replace $u$ by $1-u$ in [1]), where $\{u<1\}:=\{x \in$ $\Omega: u(x)<1\}$ and is $|\{u<1\}|$ its Lebesgue measure. One can interpret the second term in $J_{\lambda}$ as penalising the support of $(1-u)^{+}$. By reducing $\lambda$ we expect the support of $(1-u)^{+}$to grow or $D$ to shrink. When we look at (1.1), we also expect $|\nabla u|$ to decrease as $D$ shrinks. Hence the minimal $\lambda$ for which a solution exists should occur when the distance between $\partial D$ and $\partial \Omega$ becomes maximal. Therefore we expect an optimal configuration to maximise this distance, and a ball is very likely to do so. We set

$$
\Lambda_{2}(\Omega):=\inf \left\{\lambda>0: J_{\lambda} \text { has a non-trivial minimiser }\right\} .
$$

and prove that $\Lambda_{2}(\Omega) \geq \Lambda_{2}\left(\Omega^{*}\right)$, where $\Omega^{*}$ denotes the ball of same volume as $\Omega$. We also prove that equality holds if and only if $\Omega$ is a ball.

We will look at a more general problem. In [4] it is shown that for $1<p<\infty$ non-trivial minimisers of the functional

$$
J_{\lambda, p}(u):=\int_{\Omega}|\nabla u|^{p} d x+(p-1) \lambda^{p}|\{u<1\}|
$$

on $W_{0}^{1, p}(\Omega)$ solve the over-determined free boundary problem

$$
\begin{array}{rlrl}
\Delta_{p} v=0 & & \text { in } \Omega \backslash D, \\
v=0 & & \text { on } \partial \Omega, \\
v \equiv 1 & \text { on } D, \\
|\nabla v|=\lambda & \text { on } \partial D .
\end{array}
$$

Similarly as before we set

$$
\Lambda_{p}(\Omega):=\inf \left\{\lambda>0: J_{\lambda, p} \text { has a non-trivial minimiser }\right\} .
$$

First we establish the following existence result.

Theorem 1.1. The functional $J_{\lambda, p}$ has a non-trivial minimiser if and only if $\lambda \geq \Lambda_{p}(\Omega)$. Moreover, $\min J_{\lambda, p}=J_{\lambda, p}(0)$ if and only if $\lambda \leq \Lambda_{p}(\Omega)$. 
As zero is the only minimiser of $J_{0, p}(u)=\|\nabla u\|_{p}^{p}$ the above theorem implies that $\Lambda_{p}(\Omega)>0$. Our main result is the following isoperimetric inequality. The proof of the sharpness of that inequality relies in an essential way on the fact from Theorem 1.1 that zero and a nontrivial $u \in W_{0}^{1, p}(\Omega)$ both minimize $J_{\Lambda_{p}(\Omega), p}$.

Theorem 1.2. Let $\Omega$ be an arbitrary bounded domain in $\mathbb{R}^{N}$ and $\Omega^{*}$ a ball of same volume as $\Omega$. Then

$$
\Lambda_{p}(\Omega) \geq \Lambda_{p}\left(\Omega^{*}\right)
$$

with equality if and only if $\Omega$ is a ball up to a set of p-capacity zero. Moreover, if $\Omega^{*}$ has radius $r$ then

$$
\Lambda_{p}\left(\Omega^{*}\right)=\frac{p}{p-1}\left(\frac{p}{N}\right)^{(N-1) /(p-N)} \frac{1}{r}
$$

if $N \neq p$ and

$$
\Lambda_{N}\left(\Omega^{*}\right)=\frac{N}{N-1} e^{(1-1 / N)} \frac{1}{r}
$$

if $N=p$.

Note that $\Lambda_{p}\left(\Omega^{*}\right)$ is a continuous function of $p \in(1, \infty)$. Also, if $p>N$, then points have positive $p$-capacity. Hence, if $\Lambda_{p}(\Omega)=\Lambda_{p}\left(\Omega^{*}\right)$ and $p>N$, then $\Omega$ is a ball.

Remark 1.3. If the integral $\int_{\Omega}|\nabla u|^{p} d x$ in $J_{\lambda, p}(u)$ is replaced by $\int_{\Omega} G(|\nabla u|) d x$, with suitable assumptions on $G$, including convexity of $G$, one can consider a more general quasi-linear equation for functions in the appropriate Orlicz space. Details of this can be found in [12].

A conjecture related to the above theorem appears in Flucher and Rumpf [5, page 202]. The difference is that we only look at solutions of (1.4) which minimise the energy functional $J_{\lambda, p}$, whereas [5] look at all solutions, that is,

$$
\lambda_{p}(\Omega):=\inf \{\lambda>0:(1.4) \text { has a non-trivial solution }\} .
$$

A comparison of the optimal constants on the ball as computed in Section 4 reveals that $\lambda_{p}(B)<\Lambda_{p}(B)$ if $\Omega=B$ is a ball. The new result in Theorem 1.1 is that there exists a non-trivial minimiser for $\lambda=\Lambda_{p}(\Omega)$. A similar result is proved in [9, Theorem 3.1] for $\lambda=\lambda_{p}(\Omega)$ and for convex $\Omega$, but with completely different techniques to the ones we use. Also, [11] claim to prove the conjecture by Flucher and Rumpf.

Since the energy minimising solutions have attracted quite some interest with the work in [1] in case $p=2$ and [4] for general $p \in(1, \infty)$, our result should still be of interest. We give a proof of (1.6) in Section 3 and compute the optimal values in Section 4. Theorem 1.1 is proved in Section 2. 


\section{Existence of minimisers}

In this section we establish the existence results for minimisers stated in Theorem 1.1. We throughout assume that $\Omega \subset \mathbb{R}^{N}$ is a bounded open set.

Proposition 2.1. Let $J_{\lambda, p}$ and $\Lambda_{p}(\Omega)$ be defined as in the previous section.

(i) If there exists $w \in W_{0}^{1, p}(\Omega)$ such that $J_{\lambda, p}(w)<J_{\lambda, p}(0)=(p-1) \lambda^{p}|\Omega|$, then $J_{\lambda, p}$ has a non-trivial minimiser.

(ii) For $\lambda>0$ large enough $J_{\lambda, p}$ has a non-trivial minimiser.

(iii) Suppose $\mu>0$ is such that $J_{\mu, p}$ has a nontrivial minimiser $u \in W_{0}^{1, p}(\Omega)$. Then $J_{\lambda, p}$ has a non-trivial minimiser for all $\lambda>\mu$.

(iv) We have min $J_{\lambda, p}=J_{\lambda, p}(0)$ if and only if $\lambda \leq \Lambda_{p}(\Omega)$.

Proof. (i) Since $J_{\lambda, p}(u) \geq 0$ for all $u \in W_{0}^{1, p}(\Omega)$ we can choose a minimising sequence $u_{n} \in W_{0}^{1, p}(\Omega)$ with $J_{\lambda, p}\left(u_{n}\right) \rightarrow \inf _{u \in W_{0}^{1, p}(\Omega)} J_{\lambda, p}(u)$. By definition of $J_{\lambda, p}$ the sequence $\left(u_{n}\right)$ is bounded in $W_{0}^{1, p}(\Omega)$ and therefore has a subsequence $\left(u_{n_{k}}\right)$ converging weakly in $W_{0}^{1, p}(\Omega)$ and pointwise almost everywhere in $\Omega$ to some function $u$. Hence

$$
\|\nabla u\|_{p}^{p} \leq \liminf _{k \rightarrow \infty}\left\|\nabla u_{n_{k}}\right\|_{p}^{p}
$$

and by Fatou's Lemma

$$
\int_{\Omega} \chi_{\{u<1\}} d x \leq \liminf _{k \rightarrow \infty} \int_{\Omega} \chi_{\left\{u_{n_{k}}<1\right\}} d x
$$

where $\chi_{A}$ is the indicator function of a set $A \subseteq \mathbb{R}^{N}$ given by $\chi_{A}(x)=1$ if $x \in A$ and zero otherwise. By definition of $J_{\lambda, p}$ and the choice of $\left(u_{n}\right)$

$$
J_{\lambda, p}(u) \leq \liminf _{k \rightarrow \infty} J_{\lambda, p}\left(u_{n_{k}}\right)=\inf _{v \in W_{0}^{1, p}(\Omega)} J_{\mu, p}(v) .
$$

Thus, $u$ is a minimiser. It is non-trivial since by assumption $J_{\lambda, p}(u) \leq$ $J_{\lambda, p}(w)<J_{\lambda, p}(0)$.

(ii) Let $\varphi \in C_{c}^{\infty}(\Omega)$ such that $|\{\varphi \geq 1\}|>0$. Then note that

$$
J_{\lambda, p}(\varphi)-J_{\lambda, p}(\varphi)(0)=\|\nabla \varphi\|_{p}^{p}-(p-1) \lambda^{p}|\{\varphi \geq 1\}|<0
$$

for $\lambda>0$ large enough. Now apply (i). 
(iii) Clearly, if $u$ is a non-trivial minimiser of $J_{\mu, p}$, then $J_{\lambda, p}(u) \leq J_{\lambda, p}(0)$. Also, $|\{u<1\}|<|\Omega|$ since otherwise $J_{\lambda, p}(0)<J_{\lambda, p}(u)$ and $u$ is not a minimiser. Hence from the definition of $J_{\mu, p}$ we have

$$
\begin{aligned}
J_{\lambda, p}(u) & =\|\nabla u\|_{p}^{p}+(p-1) \lambda^{p}|\{u<1\}| \\
& =J_{\mu, p}(u)+(p-1)\left(\lambda^{p}-\mu^{p}\right)|\{u<1\}| \\
& \leq(p-1) \mu^{p}|\Omega|+(p-1)\left(\lambda^{p}-\mu^{p}\right)|\{u<1\}| \\
& =(p-1) \lambda^{p}|\Omega|-(p-1)\left(\lambda^{p}-\mu^{p}\right)(|\Omega|-|\{u<1\}|) .
\end{aligned}
$$

Since $|\{u<1\}|<|\Omega|$ we conclude that $J_{\lambda, p}(u)<(p-1) \lambda^{p}|\Omega|=J_{\lambda, p}(0)$ for all $\lambda>\mu$. By (i) $J_{\lambda, p}$ has a non-trivial minimiser for all $\lambda>\mu$.

(iv) If $\lambda<\Lambda_{p}(\Omega)$, then clearly $\min _{u \in W_{0}^{1, p}(\Omega)} J_{\lambda, p}(u)=J_{\lambda, p}(0)$, so assume that $\mu:=\Lambda_{p}(\Omega)$. Assume that $u$ is a minimiser of $J_{\mu, p}$ and suppose that strict inequality holds in (2.1). Then clearly $J_{\lambda, p}(u)<J_{\lambda, p}(0)=(p-1) \lambda^{p}|\Omega|$ if $\lambda<\mu$ is close enough to $\mu$. However, this contradicts the definition of $\mu=\Lambda_{p}(\Omega)$ since otherwise (i) implies the existence of a minimiser for some $\lambda<\mu$.

To prove that $J_{\lambda, p}$ also has a non-trivial minimiser for $\lambda=\Lambda_{p}(\Omega)$ we need to compare $\|\nabla u\|_{p}$ with the measure of $\{u \geq 1\}$. In the following lemma we get such an estimate. It is motivated by the estimate of the measure of a set in terms of its capacity (see e.g. [6, page 5]), but does not rely on capacity.

Lemma 2.2. Let $1<p \leq N$. Then there exist $q>p$ and $C>0$ only depending on $N, p$ and $|\Omega|$ such that $|\{u \geq 1\}| \leq C\|\nabla u\|_{p}^{q}$ for all $u \in W_{0}^{1, p}(\Omega)$.

Proof. If $1<p<N$, by the Sobolev inequality there exists a constant $C>0$ only depending on $N$ and $p$ such that

$$
|\{u \geq 1\}| \leq \int_{\Omega}|u|^{N p /(N-p)} d x \leq C\|\nabla u\|_{p}^{N p /(N-p)}
$$

for all $u \in W_{0}^{1, p}(\Omega)$. Hence we can set $q:=N p(N-p)>p$. If $p=N$ choose $p_{0} \in(N / 2, N)$ and apply the above inequality and Hölder's inequality to get

$$
|\{u \geq 1\}| \leq C\|\nabla u\|_{p_{0}}^{N p_{0} /\left(N-p_{0}\right)} \leq C|\Omega|^{\theta}\|\nabla u\|_{p}^{N p_{0} /\left(N-p_{0}\right)}
$$

for all $u \in W_{0}^{1, p}(\Omega)$, where $\theta$ is a constant depending only on $p_{0}$ and $N$. Hence we can set $q:=N p_{0} /\left(N-p_{0}\right)$. Clearly $q>N$ since $p_{0}>N / 2$.

Since by definition of $\Lambda_{p}(\Omega)$ the functional $J_{\lambda, p}$ has no non-trivial minimiser for $\lambda<\Lambda_{p}(\Omega)$ the following proposition will conclude the proof of Theorem 1.1. It is also the most original and new part of the proof. 
Proposition 2.3. If $\mu=\Lambda_{p}(\Omega)$, then $J_{\mu, p}$ has a non-trivial minimiser.

Proof. By definition of $\Lambda_{p}(\Omega)$ either there exists a non-trivial minimiser or there is a sequence $\left(\lambda_{n}\right)$ such that $\lambda_{n}>\mu$ for all $n \in \mathbb{N}, \lambda_{n} \rightarrow \mu$ and $J_{\lambda_{n}, p}$ has a non-trivial minimiser $u_{n} \in W_{0}^{1, p}(\Omega)$ for every $n \in \mathbb{N}$. Then

$$
J_{\lambda, p}\left(u_{n}\right)=\left\|\nabla u_{n}\right\|_{p}^{p}+(p-1) \lambda_{n}^{p}\left|\left\{u_{n}<1\right\}\right| \leq(p-1) \lambda_{n}^{p}|\Omega|
$$

for all $n \in \mathbb{N}$. Since $\left(\lambda_{n}\right)$ is a convergent sequence, $\left(u_{n}\right)$ is bounded in $W_{0}^{1, p}(\Omega)$. It therefore has a convergent subsequence such that $u_{n_{k}} \rightarrow u$ weakly in $W_{0}^{1, p}(\Omega)$ and pointwise almost everywhere. Fix $v \in W_{0}^{1, p}(\Omega)$. As in the proof of Proposition 2.1(i)

$$
J_{\mu, p}(u) \leq \liminf _{k \rightarrow \infty} J_{\lambda_{n_{k}}, p}\left(u_{n_{k}}\right) \leq \liminf _{k \rightarrow \infty} J_{\lambda_{n_{k}}, p}(v)=J_{\mu, p}(v)
$$

where in the second inequality we use that $u_{n_{k}}$ are minimisers for $J_{\lambda_{n_{k}}, p}$. Hence $u \in W_{0}^{1, p}(\Omega)$ is a minimiser of $J_{\mu, p}$.

To conclude the proof we need to show that $u \neq 0$. If $u=0$ and $p>N$, then $u_{n_{k}} \rightarrow 0$ uniformly as $k \rightarrow \infty$ since $W_{0}^{1, p}(\Omega)$ is compactly embedded into $C(\bar{\Omega})$. Therefore there exists $m \in \mathbb{N}$ such that $\left\|u_{m}\right\|_{\infty}<1$ and so

$$
J_{\lambda_{m}, p}\left(u_{m}\right)=\left\|\nabla u_{m}\right\|_{p}^{p}+(p-1) \lambda_{m}^{p}|\Omega|>(p-1) \lambda_{m}^{p}|\Omega|=J_{\lambda_{m}, p}(0)
$$

since by assumption $u_{m} \neq 0$. As $u_{m}$ was a non-trivial minimiser this is a contradiction, and so $u \neq 0$.

We next look at the case $1<p \leq N$. Again assume that $u=0$. Then by Rellich's theorem we have $u_{n_{k}} \rightarrow 0$ in $L_{p}(\Omega)$ and so

$$
\left|\left\{u_{n_{k}} \geq 1\right\}\right| \leq \int_{\left\{u_{n_{k}} \geq 1\right\}}\left|u_{n_{k}}\right|^{p} d x \leq\left\|u_{n_{k}}\right\|_{p}^{p} \rightarrow 0
$$

Hence (2.2) with $u=v=0$ implies that

$$
\begin{aligned}
\mu^{p}(p-1)|\Omega|=J_{\mu, p}(0) \leq \liminf _{k \rightarrow \infty} & J_{\lambda_{n_{k}}, p}\left(u_{n_{k}}\right) \\
& \leq \liminf _{k \rightarrow \infty} J_{\lambda_{n_{k}}, p}(0)=J_{\mu, p}(0)=\mu^{p}(p-1)|\Omega|,
\end{aligned}
$$

and therefore $\left\|\nabla u_{n_{k}}\right\|_{p} \rightarrow 0$. As $J_{\lambda_{n}, p}(0)=(p-1) \lambda_{n}^{p}|\Omega|$, Lemma 2.2 implies the existence of constants $C>0$ and $q>p$ such that

$$
\begin{aligned}
& J_{\lambda_{n}, p}\left(u_{n}\right)=J_{\lambda_{n}, p}(0)+\left\|\nabla u_{n}\right\|_{p}^{p}-(p-1) \lambda_{n}^{p}\left|\left\{u_{n} \geq 1\right\}\right| \\
& \geq J_{\lambda_{n}, p}(0)+\left\|\nabla u_{n}\right\|_{p}^{p}-C(p-1) \lambda_{n}^{p}\left\|\nabla u_{n}\right\|_{p}^{q} \\
& \quad=J_{\lambda_{n}, p}(0)+\left\|\nabla u_{n}\right\|_{p}^{p}\left(1-C(p-1) \lambda_{n}^{p}\left\|\nabla u_{n}\right\|_{p}^{q-p}\right)
\end{aligned}
$$


for all $n \in \mathbb{N}$. Since $\left\|\nabla u_{n_{k}}\right\|_{p} \rightarrow 0, \lambda_{n} \rightarrow \mu$ and $q>p$ there exists $m \in \mathbb{N}$ with

$$
1-C(p-1) \lambda_{m}^{p}\left\|\nabla u_{m}\right\|_{p}^{q-p}>0
$$

and hence by $(2.3)$ we get $J_{\lambda_{m}, p}\left(u_{m}\right)>J_{\lambda_{m}, p}(0)$. This is a contradiction since $u_{m}$ was assumed to be a minimiser for $J_{\lambda_{m}, p}$, and so $u \neq 0$ as claimed.

\section{Proof of the isoperimetric inequality}

This whole section is devoted to the proof of the first part of Theorem 1.2. We let $\Omega \subset \mathbb{R}^{N}$ be a bounded open set and let $\Omega^{*} \subset \mathbb{R}^{N}$ be an open ball with the same volume as $\Omega$. For $v \in W_{0}^{1, p}\left(\Omega^{*}\right)$ set

$$
J_{\lambda, p}^{*}(v)=\int_{\Omega^{*}}\left(|\nabla v|^{p} d x+(p-1) \lambda^{p}|\{v<1\}|\right.
$$

and recall that minimisers are solutions of (1.4) with $\Omega$ replaced by $\Omega^{*}$. Let $\lambda \geq \Lambda_{p}(\Omega)$. By Theorem $1.1 J_{\lambda, p}$ has a non-trivial minimiser $u \in W_{0}^{1, p}(\Omega)$. Consider its Schwarz symmetrisation $u^{*}$ (see [10] for a definition and properties). By well known properties of Schwarz symmetrisation $u^{*} \in W_{0}^{1, p}\left(\Omega^{*}\right)$, $\left\|\nabla u^{*}\right\|_{p} \leq\|\nabla u\|_{p}$ and $\left|\left\{u^{*}<1\right\}\right|=|\{u<1\}|$. Also $u^{*}$ is non-zero and

$$
\begin{aligned}
J_{\lambda, p}^{*}\left(u^{*}\right)=\left\|\nabla u^{*}\right\|_{p}^{p}+(p-1) \lambda^{p}\left|\left\{u^{*}<1\right\}\right| \\
\leq\|\nabla u\|_{p}^{p}+(p-1) \lambda^{p}|\{u<1\}|=J_{\lambda, p}(u) .
\end{aligned}
$$

In particular $J_{\lambda, p}^{*}\left(u^{*}\right) \leq J_{\lambda, p}(u) \leq J_{\lambda, p}(0)=J_{\lambda, p}^{*}(0)$. If $J_{\lambda, p}^{*}\left(u^{*}\right)<J_{\lambda, p}^{*}(0)$, then by Proposition 2.1(i) $J_{\lambda, p}^{*}$ has a non-trivial minimiser. If $J_{\lambda, p}^{*}\left(u^{*}\right)=$ $J_{\lambda, p}^{*}(0)$, then either $u^{*}$ is a non-trivial minimiser, or inf $J_{\lambda, p}^{*}<(p-1) \lambda^{p}\left|\Omega^{*}\right|$ and Proposition 2.1(i) implies the existence of a non-trivial minimiser. In any case, if $J_{\lambda, p}$ has a non-trivial minimiser, so does $J_{\lambda, p}^{*}$. Hence by definition of $\Lambda_{p}(\Omega)$ and $\Lambda_{p}\left(\Omega^{*}\right)$ the inequality (1.6) follows.

It remains to prove the sharpness of (1.6). We assume that $\Lambda_{p}(\Omega)=$ $\Lambda_{p}\left(\Omega^{*}\right)$. The aim is to show that $\Omega$ is a ball up to a set of capacity zero. To simplify notation we denote the common value of $\Lambda_{p}(\Omega)$ and $\Lambda_{p}\left(\Omega^{*}\right)$ by $\Lambda$ and let $r$ be the radius of the ball $\Omega^{*}$. By Theorem 1.1 zero is a minimiser for the problem on $\Omega$ and also on $\Omega^{*}$. Hence, using (3.1)

$$
(p-1) \Lambda^{p}|\Omega|=J_{\Lambda, p}^{*}(0) \leq J_{\Lambda, p}^{*}\left(u^{*}\right) \leq J_{\Lambda, p}(u)=J_{\Lambda, p}(0)=(p-1) \Lambda^{p}|\Omega| .
$$

We conclude that $J_{\Lambda, p}^{*}\left(u^{*}\right)=J_{\Lambda, p}(u)$. In particular, $u^{*}$ is a minimiser of $J_{\Lambda, p}^{*}$. Since there is a unique radially symmetric minimiser on $\Omega^{*}$ (see the argument at the start of Section 4) we conclude that $u^{*}$ coincides with (4.1) 
if $p \neq N$ and (4.2) if $p=N$ with $\rho$ given by (4.6) and (4.8), respectively. In particular, $\nabla u^{*}(x)=\nabla u_{\rho}(x) \neq 0$ whenever $0<u_{\rho}(x)<1=\max u_{\rho}$. Therefore, [3, Theorem 1.1] applies and so, up to translation, $u=u^{*}=u_{\rho}$ almost everywhere. Extending $u, u^{*}$ by zero outside $\Omega$ and $\Omega^{*}$, respectively we can assume that $u, u^{*} \in W^{1, p}\left(\mathbb{R}^{N}\right)$. We can then replace $u$ and $u^{*}$ by a quasi-continuous representative as defined in [8, Theorem 4.5]. Since $u_{\rho}$ is continuous and $u^{*}=u_{\rho}$ almost everywhere, $u_{\rho}$ is the quasi-continuous representative of $u^{*}$. Hence $u_{\rho}=u$ quasi everywhere, that is, except possibly on a set of $p$-capacity zero. Also, as $u \in W_{0}^{1, p}(\Omega)$ we know from [8, Theorem 4.5] that $u=0$ quasi everywhere on $\Omega^{c}$. Combining the two facts we get $u=u_{\rho}=0$ quasi-everywhere on $C:=\Omega^{*} \backslash \Omega$. Since $u_{\rho}>0$ on $\Omega^{*}$ we conclude that $C$ must have $p$-capacity zero. Hence $\Omega=\Omega^{*}$ is a ball except possibly for a set of $p$-capacity zero.

\section{The optimal constants}

In this section we look at (1.4) in case $\Omega=B_{r}$ is a ball of radius $r>0$ centred at the origin. We want to compute the value of $\Lambda_{p}\left(B_{r}\right)$. To do so we assume that $\lambda \geq \Lambda_{p}\left(B_{r}\right)$ and that $u \in W_{0}^{1, p}\left(B_{r}\right)$ is a minimiser of $J_{\lambda, p}$. Let $u^{*} \in W_{0}^{1, p}\left(B_{r}\right)$ be its Schwarz symmetrisation. According to (3.1) we have $J_{\lambda, p}\left(u^{*}\right) \leq J_{\lambda, p}(u)$. Hence there is a radially symmetric minimiser $u_{\rho}$ and we can assume without loss of generality that $u_{\rho}=u_{\rho}^{*}$. Let $\rho>0$ be the radius of the ball $\{u \geq 1\}$. By [4, Theorem 2.1] (or [1, Lemma 2.4] in case $p=2$ ) the minimiser is $p$-harmonic on $B_{r} \backslash \bar{B}_{\rho}$ with $u=0$ on $\partial B_{r}$ and $u=1$ on $\partial B_{\rho}$. As there is precisely one such $p$-harmonic function (see [8, Lemma 8.5])

$$
u_{\rho}(x)= \begin{cases}\frac{|x|^{(p-N) /(p-1)}-r^{(p-N) /(p-1)}}{\rho^{(p-N) /(p-1)}-r^{(p-N) /(p-1)}} & \text { if } \rho \leq|x| \leq r \\ 1 & \text { if } 0 \leq|x| \leq \rho\end{cases}
$$

if $p \neq N$ and

$$
u_{\rho}(x)= \begin{cases}\frac{\log |x|-\log r}{\log \rho-\log r} & \text { if } \rho \leq|x| \leq r \\ 1 & \text { if } 0 \leq|x| \leq \rho\end{cases}
$$

if $p=N$ (see $[5,9]$ ). Given $\rho \in(0, r)$ one can compute $\lambda=\left|\nabla u_{\rho}\right|$ for $|x|=\rho$, and then minimise $\lambda$. This yields the smallest possible value of $\lambda$ such that (1.4) has a non-trivial solution. These optimal values have been calculated in [5] for $p=2$ and in [9] for general $p \in(1, \infty)$. They are

$$
\lambda_{p}\left(B_{r}\right)=\frac{\left|\frac{p-N}{p-1}\right|}{r \mid\left(\frac{p-1}{N-1}\right)^{(N-1) /(N-p)}-\left(\frac{p-1}{N-1}\right)^{(p-1) /(N-p) \mid}}
$$


if $p \neq N$ and

$$
\lambda_{p}\left(B_{r}\right)=\frac{e}{r}
$$

if $p=N$. Unfortunately, the corresponding solution does not minimise $J_{\lambda_{p}, p}$. In case $p=2$ this is pointed out in [5, Section 5.3], but also follows from the calculations below. To obtain $\Lambda_{p}\left(B_{r}\right)$ we start by computing $J_{\lambda, p}\left(u_{\rho}\right)$. We first consider the case $p \neq N$. An elementary calculation yields

$$
\left|\nabla u_{\rho}(x)\right|=\left|\frac{p-N}{p-1}\right| \frac{|x|^{(1-N) /(p-1)}}{\left|\rho^{(p-N) /(p-1)}-r^{(p-N) /(p-1)}\right|}
$$

for $\rho \leq|x| \leq r$ and zero elsewhere. Because

$$
\begin{aligned}
\int_{\rho}^{r} s^{p(1-N) /(p-1)} s^{N-1} d s=\int_{\rho}^{r} s^{(p-N) /(p-1)-1} d s & \\
& =\frac{p-1}{p-N}\left(r^{(p-N) /(p-1)}-\rho^{(p-N) /(p-1)}\right)
\end{aligned}
$$

we get

$$
\int_{B_{r}}\left|\nabla u_{\rho}(x)\right|^{p} d x=\left|\frac{p-N}{p-1}\right|^{p-1} \frac{\omega_{N}}{\left|\rho^{(p-N) /(p-1)}-r^{(p-N) /(p-1)}\right|^{p-1}},
$$

where $\omega_{N}$ is the surface area of the unit sphere in $\mathbb{R}^{N}$. According to Theorem 1.1 we have to find the smallest possible $\lambda>0$ such that

$$
J_{\lambda, p}\left(u_{\rho}\right)=J_{\lambda, p}(0)=(p-1) \lambda^{p}\left|B_{r}\right|=(p-1) \frac{\omega_{N}}{N} r^{N} \lambda^{p} .
$$

Using the definition of $J_{\lambda, p}$ and $u_{\rho}$ we therefore require that

$$
\begin{aligned}
\left|\frac{p-N}{p-1}\right|^{p-1} \frac{\omega_{N}}{\mid \rho^{(p-N) /(p-1)}-r^{(p-N) /(p-1) \mid p-1}} \\
+(p-1) \lambda^{p} \frac{\omega_{N}}{N}\left(r^{N}-\rho^{N}\right)=(p-1) \frac{\omega_{N}}{N} r^{N} \lambda^{p}
\end{aligned}
$$

or equivalently

$$
N\left|\frac{p-N}{p-1}\right|^{p-1}=(p-1) \lambda^{p} \rho^{N}\left|\rho^{(p-N) /(p-1)}-r^{(p-N) /(p-1)}\right|^{p-1} .
$$

Clearly we get the smallest value of $\lambda$ if we pick $\rho \in(0, r)$ such that

$$
\rho^{N}\left|\rho^{(p-N) /(p-1)}-r^{(p-N) /(p-1)}\right|^{p-1}
$$


is maximal, and then compute the corresponding value of $\lambda$ from (4.5). An elementary calculation shows that this is the case for

$$
\rho=\left(\frac{N}{p}\right)^{(p-1) /(p-N)} r
$$

and hence, if we substitute that value of $\rho$ into (4.5), then

$$
\Lambda_{p}\left(B_{r}\right)=\frac{p}{p-1}\left(\frac{p}{N}\right)^{(N-1) /(p-N)} \frac{1}{r}
$$

We could confirm the above by computing $\left|\nabla u_{\rho}\right|$ for the above value of $\rho$. If $p=N$ we proceed in exactly the same way to get

$$
\rho=e^{-(1-1 / N)} r
$$

and

$$
\Lambda_{N}\left(B_{r}\right)=\frac{N}{N-1} e^{(1-1 / N)} \frac{1}{r}
$$

It is also evident that

$$
\left(\frac{p}{N}\right)^{(N-1) /(p-N)}=\left(1+\frac{\frac{1}{N}}{\frac{1}{p-N}}\right)^{(N-1) /(p-N)} \rightarrow e^{(N-1) / N}
$$

as $p \rightarrow N$, so $\Lambda_{p}\left(B_{r}\right) \rightarrow \lambda_{N}\left(B_{r}\right)$ as $p \rightarrow N$. Also note that $\lambda_{p}\left(B_{r}\right)<\Lambda_{p}\left(B_{r}\right)$ for all $p \in(1, \infty)$. In particular, this proves the second part of Theorem 1.2.

Acknowledgement B.K. thanks Henrik Shahgholian for pointing out this problem during the open problem session of the ESF-sponsored conference "FBP 2008" in Stockholm. D.D. thanks for a very pleasant visit to the University of Cologne.

\section{References}

[1] H. W. Alt and L. A. Caffarelli, Existence and regularity for a minimum problem with free boundary, J. Reine Angew. Math. 325 (1981), 105-144.

[2] A. Beurling, On free-boundary value problems for the Laplace equation, Seminars on analytic functions (Princeton 1957), vol. I, Institute for Advanced Study, Princeton, 1958, pp. 248-263.

[3] J. E. Brothers and W. P. Ziemer, Minimal rearrangements of Sobolev functions, J. Reine Angew. Math. 384 (1988), 153-179. 
[4] D. Danielli and A. Petrosyan, A minimum problem with free boundary for a degenerate quasilinear operator, Calc. Var. Partial Differential Equations 23 (2005), 97-124.

[5] M. Flucher and M. Rumpf, Bernoulli's free-boundary problem, qualitative theory and numerical approximation, J. Reine Angew. Math. 486 (1997), 165-204.

[6] J. Frehse, Capacity methods in the theory of partial differential equations, Jahresber. Deutsch. Math.-Verein. 84 (1982), 1-44.

[7] K. Friedrichs, Über ein Minimumproblem für Potentialströmungen mit freiem Rande, Math. Ann. 109 (1934), 60-82.

[8] J. Heinonen, T. Kilpeläinen, and O. Martio, Nonlinear potential theory of degenerate elliptic equations, Oxford Mathematical Monographs, Clarendon Press, New York, 1993.

[9] A. Henrot and H. Shahgholian, Existence of classical solutions to a free boundary problem for the p-Laplace operator. II. The interior convex case, Indiana Univ. Math. J. 49 (2000), 311-323.

[10] B. Kawohl, Rearrangements and convexity of level sets in PDE, Lecture Notes in Mathematics, vol. 1150, Springer-Verlag, Berlin, 1985.

[11] I. Ly and D. Seck, Isoperimetric inequality for an interior free boundary problem with p-Laplacian operator, Electron. J. Differential Equations 2004 (2004) no. 109, 1-12.

[12] S. Martínez and N. Wolanski, A minimum problem with free boundary in Orlicz spaces, Adv. Math. 218 (2008), 1914-1971.

Author's addresses:

Daniel Daners

School of of Mathematics and Statistics

The University of Sydney, NSW 2006, Australia

daniel.daners@sydney .edu.au

and

Bernd Kawohl

Mathematisches Institut

Universität zu Köln

D-50923 Köln, Germany

kawohl@mi . uni-koeln.de 